\title{
CORRELACION ENTRE EL HETEROMORFISMO SOMATICO \\ Y LA RESPUESTA GERMINATIVA DE SEMILLAS \\ DE MASTICHODENDRON FOETIDISSIMUM (JACQ.) CRONQ.
}

\author{
JoRge A. SANCHEZ \\ BARBARA MUÑOZ \\ RAMON ORTA \\ ERIC CaLVo \\ Ricardo Herrera \\ Instituto de Ecología y Sistemática \\ Ministerio de Ciencia, Tecnología y Medio Ambiente \\ Carretera de Varona km 3.5 \\ Capdevila, Boyeros \\ Apartado postal 8010 \\ Código postal 10800 \\ Habana 8, Cuba
}

\begin{abstract}
RESUMEN
Mastichodendron foetidissimum (Jacq.) Cronq. es un árbol con semillas heteromórficas que difieren en forma y tamaño en función de su cuantía (1, 2 ó 3 ) en cada fruto. En este trabajo se determina la correlación existente entre esta variabilidad y su respuesta germinativa. Las semillas fueron clasificadas en tres categorías de forma (morfos I, II y III), que corresponden al número con el que se presentan en el fruto. A cada morfo se le determinaron: longitud, anchura, grosor, peso seco, peso fresco, contenido en humedad y porcentaje de germinación final. Todos los parámetros difieren entre morfos salvo el contenido en humedad. Se observa una clara relación entre las diferencias en peso de las semillas y su respuesta germinativa: las más pesadas (morfo I) presentaron los mayores porcentajes de germinación.
\end{abstract}

\section{ABSTRACT}

Mastichodendron foetidissimum (Jacq.) Cronq. is a tree characterized by heteromorphic seeds, which differ greatly in shape and size as a result of their number $(1,2$ or 3$)$ in each fruit. In this paper the correlation between this variability and the germinative response of the seeds was determined. The seeds were classified into three categories, according to their shape (morphs I, II and III), corresponding to the number of seeds per fruit. For each morph, length, width, thickness, fresh weight, dry weight, content of humidity and percent of germination were determined. All the parameters differ between morphs, except the humidity content. The morphological variability shows a clear correlation with the germinative response: the heavier seeds (morph I) display the larger percentages of germination.

\section{INTRODUCCION}

Mastichodendron foetidissimum (Jacq.) Cronq. es un árbol de la familia Sapotaceae, que mide hasta 25 m de altura (León y Alain, 1957), muy común en toda la isla de Cuba, en bosques semideciduos cercanos a las costas altas y secas (Roig, 1975; Bisse, 1988). 
El fruto lo ingieren los animales (León y Alain, 1957; Roig, 1975) y se presenta como una baya amarilla con semillas que difieren en forma y tamaño entre frutos, hecho que se conoce como heteromorfismo somático o polimorfismo de las semillas. Este fenómeno está condicionado genéticamente y se caracteriza por la producción en una misma planta de dos o más tipos de semillas que pueden diferir totalmente en forma, tamaño y comportamiento ecofisiológico, en lo que respecta a dispersión, latencia y germinación (Venable, 1985).

Para M. foetidissimum, conocido vulgarmente como "jocuma", sólo se han definido en nuestro país algunos requerimientos de la germinación de las semillas (A. Peña, comun. pers.), pero nunca se ha estudiado la correlación que puede existir entre el heteromorfismo de las mismas y su comportamiento germinativo. Este aspecto debe considerarse si se pretende tener éxito en la reforestación con árboles de dicho taxon, de alto valor maderable (Pérez y Rodríguez, 1986), debido a que el heteromorfismo suele jugar un papel importante en el establecimiento y regeneración de las especies que ocupan ambientes impredecibles o que están sujetas a cambios abióticos (Black, 1958; Baker, 1974; Cideciyan y Malloch, 1982; Philipupillai y Ungar, 1984).

El objetivo del presente trabajo es determinar la correlación existente entre la variabilidad en forma y tamaño de las semillas de $M$. foetidissimum y su conducta germinativa ante diferentes temperaturas del substrato.

\section{MATERIALES Y METODOS}

Las semillas de M. foetidissimum fueron colectadas de frutos maduros en los árboles en diciembre de 1991, en tres parcelas del Instituto de Ecología y Sistemática. Los frutos se despulparon y secaron en la sombra durante 72 horas y se almacenaron hasta su uso a $25 \pm 1^{\circ} \mathrm{C}$. Las semillas se separaron en tres categorías de forma (morfo I, II y III) que corresponden al número de semillas por fruto (Fig. 1).

Parámetros morfológicos

Se tomaron 100 semillas al azar del morfo I y del II, y 73 del morfo III (poco frecuente en la naturaleza); a éstas se les midieron los siguientes parámetros morfológicos: longitud $(\mathrm{mm})$, anchura $(\mathrm{mm})$, grosor $(\mathrm{mm})$, peso fresco $(\mathrm{g})$, peso seco $(\mathrm{g})$ y contenido de humedad (\%); el peso seco y el contenido en humedad se determinaron mediante el secado de las semillas durante 48 horas en una estufa a $80^{\circ} \mathrm{C}$.

Para determinar el índice del estado nutricional de las semillas (INS) se utilizó el peso relativo de las mismas sin cubierta, que estimó la acumulación de nutrientes del embrión y sus reservas, mediante la siguiente fórmula:

$$
\text { INS }=\frac{\text { Peso de la semilla sin cubierta }(\mathrm{g})}{\text { Peso fresco de la semilla }(\mathrm{g})} \times 100
$$

Con tal objetivo se tomaron muestras de 100 semillas para los morfos I y II, que fueron procesadas independientemente para determinar su peso con y sin cubierta. 

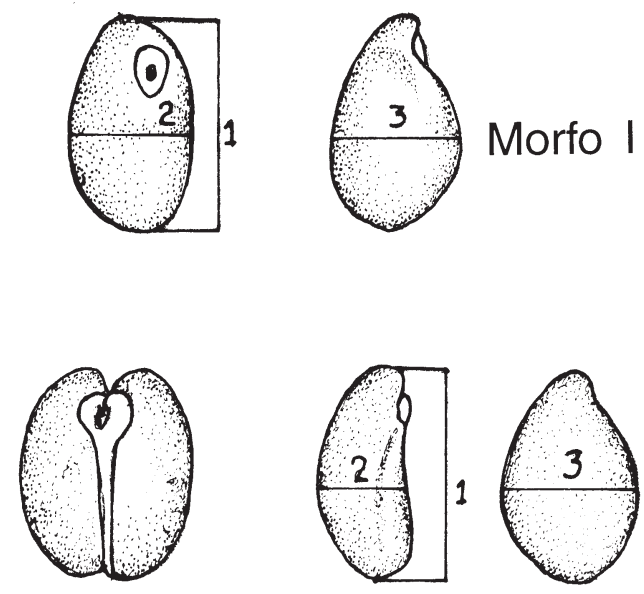

Morfo II
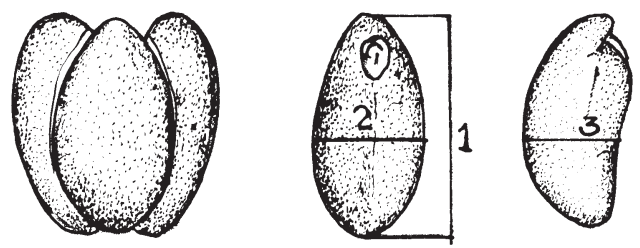

\section{Morfo III}

Fig. 1. Representación esquemática de los tipos de semillas de M. foetidissimum, en la que se observan las tres medidas tomadas: 1 (longitud), 2 (anchura) y 3 (grosor). Morfo I: presente en frutos con una semilla, Morfo II: presente en frutos con dos semillas y Morfo III: presente en frutos con tres semillas.

Pruebas de germinación

Para tal finalidad sólo se utilizaron semillas de los morfos I y II. Se diseñó un experimento trifactorial para conocer el efecto de la temperatura del substrato sobre la germinación de ambos morfos, con diferentes tratamientos pregerminativos. El gradiente de temperatura utilizado fue: 25,30 y $40^{\circ} \mathrm{C}$ y los tratamientos pregerminativos fueron: semillas sin escarificar (control) y semillas escarificadas con ácido sulfúrico a $50 \%$ durante cinco minutos (Anónimo, 1983).

Se definieron cinco réplicas de cada morfo con 20 semillas cada una por tratamiento y éstas se sembraron en placas de Petri de $12 \mathrm{~cm}$ de diámetro, en arena sílice estéril, y se regaron todos los días. Las semillas antes de sembrarse se desinfectaron en solución acuosa de bicloruro de mercurio $(0.1 \% \mathrm{P} / \mathrm{V})$ durante 10 minutos y posteriormente se enjuagaron con agua destilada estéril. El conteo de germinación se efectuó diariamente durante tres meses y se determinó el porcentaje de germinación final para cada tratamiento. 
El porcentaje de semillas vivas no germinadas y de las muertas se determinó mediante la prueba de TZ (solución acuosa al $0.01 \%$ P/V de cloruro de 2,3,5 trifenil tetrazolium, durante 24 horas a $30^{\circ} \mathrm{C}$ ).

Banco de plántulas

En el mes de diciembre del mismo año, se escogieron al azar las áreas de muestreo cercanas a las zonas iniciales de colecta de semillas. Se fijaron seis áreas de $1 \mathrm{~m}^{2}$ cada una (tres a la sombra y tres al sol), que se limpiaron de semillas viejas. Ocho meses más tarde (agosto) se determinó la cantidad de plántulas de $M$. foetidissimum por metro cuadrado presentes en el suelo así como el tipo de semillas de las que procedían.

Los parámetros morfológicos fueron estudiados por medio de un análisis de varianza de clasificación simple. Los datos de porcentaje de germinación final se transformaron al algoritmo arc sen $\sqrt{\%}$, y se procesaron mediante un análisis de clasificación simple que involucra un arreglo factorial de los tratamientos (tipo de semilla $x$ tratamiento pregerminativo).

\section{RESULTADOS Y DISCUSION}

Parámetros morfológicos

Todos los parámetros morfológicos difieren significativamente entre los morfos (Cuadro 1), salvo el contenido en humedad de las semillas.

La mayor variabilidad entre morfos se presentó para el peso fresco, el peso seco y el ancho de las semillas, lo cual indica que las diferencias en tallas y pesos entre morfos, reflejan principalmente las variaciones entre semillas en cuanto al peso del embrión con sus reservas (Cuadro 2).

Cuadro 1. Medias $(\bar{x})$ y desviación estándar (DE) de peso fresco (PF), peso seco (PS), longitud (L), anchura $(A)$, grosor $(\mathrm{G})$ y contenido en humedad $(\mathrm{CH})$, en los diferentes morfos de semillas de M. foetidissimum.

\begin{tabular}{|c|c|c|c|c|c|c|}
\hline \multirow[t]{2}{*}{ Caracteres } & \multicolumn{2}{|c|}{ Morfo I } & \multicolumn{2}{|c|}{ Morfo II } & \multicolumn{2}{|c|}{ Morfo III } \\
\hline & $\bar{x}$ & $\mathrm{DE}$ & $\bar{x}$ & $\mathrm{DE}$ & $\bar{x}$ & DE \\
\hline$P F(g)$ & $0.89 a$ & 0.13 & $0.79 b$ & 0.10 & $0.75 c$ & 0.02 \\
\hline PS (g) & $0.80 a$ & 0.03 & $0.71 b$ & 0.09 & $0.67 \mathrm{c}$ & 0.10 \\
\hline$L(\mathrm{~mm})$ & $15.12 b$ & 0.10 & $16.10 \mathrm{a}$ & 0.09 & $16.30 \mathrm{a}$ & 0.08 \\
\hline$A(\mathrm{~mm})$ & $10.41 a$ & 0.50 & $7.74 \mathrm{c}$ & 0.62 & $8.44 b$ & 0.60 \\
\hline $\mathrm{G}(\mathrm{mm})$ & $11.32 b$ & 0.10 & $11.70 \mathrm{a}$ & 0.88 & $11.33 b$ & 0.20 \\
\hline $\mathrm{CH}(\%)$ & $11.65 a$ & 0.26 & $11.50 \mathrm{a}$ & 0.36 & $11.60 \mathrm{a}$ & 0.45 \\
\hline
\end{tabular}

Medias con letras distintas en la misma línea difieren entre sí significativamente a $\mathrm{P}<0.05$ por la prueba de Duncan. 
La germinación de estas semillas morfológicamente desiguales debe dar lugar a plántulas con recursos iniciales distintos para comenzar a crecer, y por consiguiente, con diferentes posibilidades de supervivencia y establecimiento en ambientes heterogéneos o sujetos a cambios (Janzen, 1977b).

Esta variabilidad en los pesos de las semillas también puede estar relacionada con la dispersión de las mismas o de los frutos, debido a que teóricamente podrían recorrer distancias disímiles en comparación con lo que ocurriría con semillas o frutos de pesos más constantes (Janzen, 1977a; 1978).

Por último, no podemos descartar la posibilidad de que la variabilidad entre morfos esté relacionada con algún mecanismo de escape a la predación (Puchet y Vázquez-Yanes, 1987) o con la conducta germinativa de la especie. Este último aspecto se discute a continuación.

Cuadro 2. Medias $(\bar{x})$ y desviación estándar (DE) de peso fresco (PF), peso de las semillas sin cubiertas (PSC), e índice nutricional de las semillas (INS), en los morfos I y II de semillas de M. foetidissimum.

\begin{tabular}{|c|c|c|c|c|c|}
\hline \multirow[t]{2}{*}{ Caracteres } & \multicolumn{2}{|c|}{ Morfo I } & \multicolumn{2}{|c|}{ Morfo II } & \multirow[t]{2}{*}{$t$} \\
\hline & $\bar{x}$ & $\mathrm{DE}$ & $\bar{x}$ & $\mathrm{DE}$ & \\
\hline $\mathrm{PF}(\mathrm{g})$ & 0.90 & 0.12 & 0.81 & 0.07 & $\star *$ \\
\hline PSC (g) & 0.48 & 0.06 & 0.39 & 0.03 & $* * *$ \\
\hline INS (\%) & 53.37 & 2.33 & 47.91 & 2.85 & $* * *$ \\
\hline
\end{tabular}

Pruebas de germinación

En nuestras condiciones de laboratorio la temperatura del substrato jugó un papel fundamental en la conducta germinativa de las semillas recién colectadas de $M$. foetidissimum, pues sólo se obtuvo germinación a $40^{\circ} \mathrm{C}$, tanto para las escarificadas como para las no escarificadas (Cuadro 3). Por otro lado, sin embargo, cabe advertir que tal temperatura afecta la capacidad de las semillas menos vigorosas del lote (que presentó una viabilidad inicial potencial de $82.5 \%$, determinado mediante una prueba de TZ) y el máximo porcentaje de germinación alcanzado en el experimento fue de $62.5 \%$.

El efecto combinado de la temperatura $\left(40^{\circ} \mathrm{C}\right)$ con la escarificación, resultó ser un tratamiento adecuado para semillas recién colectadas de esta especie, que eliminó la impermeabilidad de las cubiertas y cualquier tipo de dormancia morfológica que pudiera existir (Anónimo, 1983; Nikolaeva, 1982; Nikolaeva et al., 1985). El tratamiento con ácido sulfúrico logró, además, acelerar la germinación y aumentar significativamente su proporción en ambos morfos (Cuadro 3). Un porcentaje superior de germinación final se registró en las semillas del morfo I, las que presentaron a su vez mayor peso fresco y un mejor estado nutricional (Cuadro 2). De esta manera, se puede afirmar que en $M$. foetidissimum existe una diversidad de comportamiento germinativo asociado al heteromorfismo de talla o peso de las semillas. 
Las temperaturas fijas de 25 y $30^{\circ} \mathrm{C}$ resultaron ser inadecuadas para la germinación y viabilidad de esta especie $-100 \%$ de semillas muertas-, debido posiblemente al efecto combinado de tales temperaturas con la entrada de agua a las semillas que, de acuerdo con Bewley y Black (1985); acelera la respiración e impide la continuación del proceso de postmaduración. Según estos mismos autores, las especies que presentan dormancia morfológica requieren durante la postmaduración de bajos contenidos de agua, relativamente altas temperaturas y aereación suficiente, aspectos que se ven afectados en nuestras condiciones de siembra. Nikolaeva (1982) plantea la necesidad de un periodo seco, para eliminar este tipo de dormancia.

Cuadro 3. Valores promedio del porcentaje de germinación final (GF) y del día de inicio de la germinación (IG) en los morfos de semillas de $M$. foetidissimum sembradas a $40^{\circ} \mathrm{C}$, con o sin previa escarificación.

\begin{tabular}{lcccc}
\hline Caracteres & \multicolumn{2}{c}{ Con escarificación } & \multicolumn{2}{c}{ Sin escarificación } \\
& Morfo I & Morfo II & Morfo I & Morfo II \\
\hline \multirow{2}{*}{ GF (\%) } & $62.0 \mathrm{a}$ & $43.3 \mathrm{~b}$ & $4.0 \mathrm{c}$ & $2.1 \mathrm{c}$ \\
IG (días) & $8.3 \mathrm{a}$ & $20.5 \mathrm{~b}$ & $20.3 \mathrm{~b}$ & $30.5 \mathrm{c}$ \\
\hline
\end{tabular}

Medias con letras diferentes en la misma fila, difieren significativamente a $\mathrm{P}<0.05$ por una prueba de Duncan.

Las semillas de $M$. foetidissimum llegan al suelo en el inicio de la estación desfavorable -época de frío o de sequía- y por consiguiente, la dormancia combinada que presentan -impermeabilidad de cubiertas e insuficiente desarrollo morfológico del embriónles permitirá sobrevivir hasta la próxima estación favorable, cuando estarán completamente maduras o listas para germinar.

La estratificación en caliente $\left(40^{\circ} \mathrm{C}\right)$ resulta ser la mejor vía para acelerar el proceso de postmaduración. Resultado similar se obtuvo para Tectona grandis (Peña et al., en prensa) y para Roystonea regia (Muñoz et al., 1992), lo que demuestra una vez más la necesidad de dicho tratamiento para algunas especies tropicales como vía para eliminar cualquier tipo de dormancia en las semillas (Nikolaeva, 1977; Nikolaeva et al., 1985).

Banco de plántulas

En los cuadros situados al sol no aparecieron plántulas al cabo de ocho meses después de la fructificación. Al parecer, bajo condiciones de altas temperaturas, éstas no logran establecerse, aunque se elimine el estado latente de las semillas. Sin embargo, en los cuadros que están ubicados a la sombra, se observó un gran número de plántulas: $60.3 \%$ correspondientes a semillas del morfo II y $39.7 \%$ a las del morfo I. Esto nos hace pensar que las semillas del morfo II al ser más frecuentes en condiciones naturales que las semillas del morfo I, podrían asegurar la germinación y establecimiento de la especie cuando las condiciones ambientales sean más favorables para estos procesos.

A su vez las semillas del morfo I, al estar en mejor estado nutricional, podrían asegurar la germinación y establecimiento en condiciones ambientales más adversas. 
Todo lo anterior indica que el polimorfismo germinativo que presenta $M$. foetidissimum, le permitirá sobrevivir y adaptarse en ambientes fluctantes y/o altamente competitivos, como corresponde a las especies rezagadas, que son las encargadas de asegurar la regeneración y el establecimiento en la última fase sucesional de los bosques semideciduos de Cuba (Torres et al., 1990).

\section{LITERATURA CITADA}

Anónimo, 1983. Manual de semillas. Facultad de Ingeniería Forestal. Centro Universitario de Pinar del Río. Pinar del Río, Cuba. 92 pp.

Baker, H. G. 1974. The evolution of weeds. Ann. Rev. Ecol. Syst. 5: 1-24.

Bewley, J. D. y M. Black 1985. Seeds. Physiology of development and germination. Plenum Press. Nueva York y Londres. $367 \mathrm{pp}$.

Bisse, J. 1988. Arboles de Cuba. Editorial Científico-Técnica. La Habana. 384 pp.

Black, J. N. 1958. Competition between plants of different initial seed sizes in swards of subterranean clover (Trifolium subterraneum L.) with particular reference to leaf area and the light microclimate. Austral. J. Agric. Res. 9: 299-318.

Cideciyan, M. A. y A. J. Malloch. 1982. Effects of size seed on the germination growth and competitive ability of Rumex crispus and Rumex obtusifolius. J. Ecol. 70: 227-232.

Janzen, D. H. 1977a. Variation in seed size within a crop of a Costa Rican Mucuna andreana (Leguminosae). Amer. J. Bot. 64: 347-349.

Janzen, D. H. 1977b. Variation in seed weight in a Costa Rican Cassia grandis (Leguminosae). Trop. Ecol. 18: $177-186$.

Janzen, D. H. 1978. Inter and intra-crop variation in seed weight of Costa Rican Ateleia herbertii Pitt. (Leguminosae). Brenesia 14/15: 311-323.

León, Hno. y Alain, Hno. 1957. Flora de Cuba. Contrib. Ocas. Mus. Hist. Nat. Colegio de la Salle 4 (16): 124-125.

Muñoz, B., R. Orta y E. Medero. 1992. Algunos aspectos de la germinación de las semillas de Roystonea regia (H.B.K.) O. F. Cook I. c. var. regia. Cien. Biol. 24: 119-123.

Nikolaeva, M. G. 1977. Algunos resultados de la dormancia de semillas (en ruso). Botanicheskii Zhurnal 2(9): 1350-1358.

Nikolaeva, M. G. 1982. Dormancia de las semillas. In: Prokofiev A. A. (ed.). Fisiología de las semillas. Cap. 4 (en ruso). Nauka. Moscú. 317 pp.

Nikolaeva, M. G., M. V. Rasumova y V. N. Gladkova 1985. Manual de técnicas pregerminativas para semillas dormantes (en ruso). Nauka. Moscú. 348 pp.

Peña, A., J. Montalvo, L. Sordo y E. Castillo (en prensa). La estraficación en caliente una solución para la germinación de Tectona grandis. Rev. Baracoa.

Pérez, J. y J. M. Rodríguez 1986. Estudio de las propiedades físicas y mecánicas de Mastichodendron foetidissimum (Jacq.) Cronq. Tesis de diploma. Centro de Investigaciones Forestales. Ciudad de la Habana. 47 pp.

Philipupillai. J. y I. A. Ungar 1984. The effect of seed dimorphism on the germination and survival of Salicornia europaea L. populations. Amer. J. Bot. 71(4): 542-549.

Puchet, C. E. y C. Vázquez-Yanes. 1987. Heteromorfismo críptico en las semillas recalcitrantes de tres especies arbóreas de la selva tropical húmeda de Veracruz, México. Phytologia 62: 100106.

Roig, J. T. 1975. Diccionario botánico de nombres vulgares cubanos. 4a. ed. Tomo 1. Editorial Pueblo y Educación. La Habana.

Torres, Y., R. A. Herrera, E. G. Cañizares, O. Valdés-Lafont, R. P. Capote, F. Delgado, F. Cejas y P. Herrera. 1990. Bases ecotecnológicas para la silvicultura tropical en Cuba. IV-Habilidades competitivas y reproductivas en especies tropicales. Instituto de Ecología y Sistemática. Ciudad de la Habana. $11 \mathrm{pp}$.

Venable, D. L. 1985. The evolutionary ecology of seed heteromorphism. Amer. Nat. 126: 577-595. 